\title{
Sequential state discrimination with quantum correlation *
}

\author{
Jin-Hua Zhang • Fu-Lin Zhang* • \\ Mai-Lin Liang
}

Received: date / Accepted: date

\begin{abstract}
The sequential unambiguous state discrimination (SSD) of two states prepared in arbitrary prior probabilities is studied, and compared with three strategies that allow classical communication. The deviation from equal probabilities contributes to the success in all the tasks considered. When one considers at least one of the parties succeeds, the protocol with probabilistic cloning is superior to others, which is not observed in the special case with equal prior probabilities. We also investigate the roles of quantum correlations in SSD, and show that the procedure requires discords but rejects entanglement. The left and right discords correspond to the part of information extracted by the first observer and the part left to his successor respectively. Their relative difference is extended by the imbalance of prior probabilities.
\end{abstract}

Keywords Sequential state discrimination · Entanglement · Discord

\section{Introduction}

The roles of quantum correlations in quantum information procedures is a fundamental problem in quantum information. These correlations have been widely investigated in various perspectives such as quantum entanglement [1], Bell nonlocality 2, and quantum discord 3, 4. One of the interesting findings in this field is that the algorithm for deterministic quantum computation with one qubit (DQC1) can surpass the performance of the corresponding classical

\footnotetext{
* Quantum Information Processing (2018) 17:260

* Corresponding author: flzhang@tju.edu.cn

Jin-Hua Zhang

Department of Physics, Xinzhou Teacher's University, Xinzhou 034000, China

School of Mathematical Science, Capital normal university, Beijing 100048, China

Fu-Lin Zhang · Mai-Lin Liang

Department of Physics, School of Science, Tianjin University, Tianjin 300072, China
} 
algorithm in the absence of entanglement between the control qubit and a completely mixed state [5,6. Thus, the entanglement which had been regarded as the only resource for demonstrating the superiority of quantum information processing [1],7 is considered to be completely unnecessary [8. The quantum discord, which gives a measurement of the nonclassical correlations and can exist in a separable state, is considered to be the key resource in this quantum algorithm and has gained wide attention [9, 10.

Another example aided by quantum discord rather than entanglement is the procedure of unambiguous state discrimination assisted by an auxiliary qubit [1, 12, . Unambiguous discrimination among linearly independent nonorthogonal quantum states is a fundamental subject in quantum information theory [13,14,15, 16, 17. In its simplest form, Alice prepares a qubit in one of two known nonorthogonal states, $\left|\Psi_{1}\right\rangle$ and $\left|\Psi_{2}\right\rangle$, and sends it to the observer Bob. Bob's task is to determine the state he received with no error permitted. The measurement has three possible outcomes, $\left|\Psi_{1}\right\rangle,\left|\Psi_{2}\right\rangle$, and failure, in which the last one is the price to pay for no error. This is realized by a positive-operator-valued measurement (POVM) on the qubit, which requires a three dimensional Hilbert space [18. The Hilbert space can be extended via either the tensor product extension or the direct sum extension [19,20, 21. The former is necessary when the dimension of the measured system is fixed, e. g. a qubit realized by a spin-half particle. In such cases, Bob has to introduce an ancillary system to couple with the principal one. This prompts the researchers 11,12] to study the quantum correlations (entanglement and discord) created in the discrimination process.

The work 8 goes even further, studying the quantum correlations in sequential state discrimination (SSD) presented in 22. In the protocol of SSD, another observer Charlie will also perform an unambiguous discrimination measurement on the same qubit after Bob's measurement. It is one of the theories to extract information from a quantum system by multiple observers [22, 23,24 . The results in 8 , demonstrate that the entanglement is not only unnecessary for Bob's recognition, but also an obstacle for the next observer Charlie. The left discord of the state in Bob's hands corresponds to the information he extracts, and the right one to the information he left to Charlie.

However, both the researches 8 and 22 have been limited to the special case with equal prior probabilities. There are some critical reasons for solving the general problem with arbitrary priors. The optimal solution to an equalprior problem often has a symmetric form. We can check the robustness of optimal solution against variations of the priors around $1 / 2$ through a general non-uniform prior result. In both the probabilistically cloning of two pure states [25] and sequential mixed states discrimination [26, initial states prepared with general non-uniform prior have demonstrated great significance. Thus, the present study will complete the results in the general case with arbitrary probabilities and check whether the existing conclusions in 8 hold.

In the next section, we give the details of SSD with arbitrary prior probabilities. We show the absence of entanglement is required for SSD. It is compared with other three protocols that allows classical communication in Sec. 3 . The 


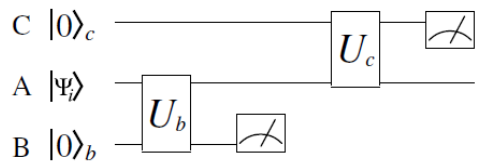

Fig. 1 Protocol for SSD. Alice has a qubit $A$ prepared in one of the two nonorthogonal states $\left|\Psi_{1}\right\rangle$ and $\left|\Psi_{2}\right\rangle$ with prior probabilities $P_{1}, P_{2}$ respectively. After the qubit is sent to Bob, a joint unitary operation is performed between the qubit $A$ and an auxiliary qutrit $B$, followed by a von Neumann measurement on the qutrit. The state discrimination is successful if the outcome is 1 (for $\left|\Psi_{1}\right\rangle$ ) and 2 (for $\left|\Psi_{2}\right\rangle$ ), but unsuccessful if the outcome is 0 . Then the qubit in the postmeasurement state is sent to Charlie by Bob. Charlie performs a similar joint unitary operation $U_{c}$ between it and his qutrit $C$ and then makes an optimal unambiguous discrimination measurement [22] on $C$.

roles of quantum correlations are discussed in Sec. 4. And the final section is a summary.

\section{Sequential state discrimination}

We now consider the procedure of SSD, which is shown in Fig 1, A qubit $A$ is prepared randomly by Alice in a state $\left|\Psi_{i}\right\rangle$ with prior probabilities $P_{i}$, where $i=1,2$, and $P_{1}+P_{2}=1$. Without loss of generality, we take the overlap $s=\left\langle\Psi_{1} \mid \Psi_{2}\right\rangle$ to be a real number $(0 \leq s \leq 1)$ and $P_{1} \in(0,1 / 2]$ in the present work. Alice sends the qubit to Bob. After performing a joint unitary transformation $U_{b}$ between $A$ and his auxiliary qutrit $B$, Bob obtains the state of the composite system as

$$
U_{b}\left|\Psi_{i}\right\rangle\left|0_{b}\right\rangle=\sqrt{q_{i}^{b}}\left|\chi_{i}\right\rangle|0\rangle_{b}+\sqrt{1-q_{i}^{b}}\left|\phi_{i}\right\rangle|i\rangle_{b},
$$

where $\left\{|0\rangle_{b},|1\rangle_{b},|2\rangle_{b}\right\}$ is a set of basis of the ancilla, and $\left|\chi_{i}\right\rangle$ and $\left|\phi_{i}\right\rangle$ are pure states of $A$. Then, Bob performs a von Neumann measurement on the qutrit with respect to the basis. He succeeds in discrimination if the ancilla collapses to $|1\rangle_{b}$ or $|2\rangle_{b}$, while he fails if the outcome is $|0\rangle_{b}$. The average success probability of Bob can be obtained as

$$
P_{b}=P_{1}\left(1-q_{1}^{b}\right)+P_{2}\left(1-q_{2}^{b}\right) .
$$

The inner product is conserved under the unitary operation. Thus, the states $\left|\chi_{i}\right\rangle$ satisfy the constraint $\sqrt{q_{1}^{b} q_{2}^{b}}\left\langle\chi_{1} \mid \chi_{2}\right\rangle=s$. Here, we denote the overlap $\left\langle\chi_{1} \mid \chi_{2}\right\rangle=t$, with $s \leq t \leq 1$. The change from $s$ to $t$ corresponds to the information Bob extracts from the qubit $A$ in his measurement 8,22 . When $t=s$, the overlap constraint demands $q_{1}^{b}=q_{2}^{b}=1$, which leads to the success probability to be zero. When $t=1$, in our following discussion, one can find that the discrimination of the next observer has a zero success probability. That is, all the information encoded in qubit $A$ is extracted by Bob. 


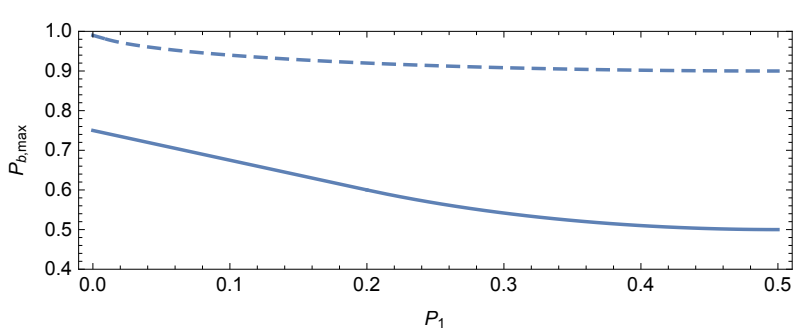

Fig. 2 The optimal success probability $P_{b, \max }$ as a function of the parameter $P_{1}$ for $s=$ $0.05, t=0.06$ (solid line), 0.1 (dotted line).

For fixed values of $s$ and $t$, the success probability $P_{b}$ of Bob can be maximized into two forms as

$$
\begin{array}{ll}
\text { (i) : } P_{b, \max }=1-2 \sqrt{P_{1} P_{2}} \frac{s}{t}, & \text { when } \frac{s^{2}}{s^{2}+t^{2}} \leq P_{1} \leq \frac{1}{2} \\
\text { (ii) : } P_{b, \max }=P_{2}\left(1-\frac{s^{2}}{t^{2}}\right), & \text { when } 0<P_{1}<\frac{s^{2}}{s^{2}+t^{2}} .
\end{array}
$$

In the above two cases, the values of the $q_{1}^{b}$ corresponding to the optimal success probabilities are (i): $q_{1}^{b}=\sqrt{P_{2} / P_{1}} s / t$ and (ii): $q_{1}^{b}=1$. When $q_{1}^{b}=1$, the state $|1\rangle_{b}$ of $B$ is absent in Eq. (10), and simultaneously the first term in Eq. (2) vanishes. That is, Bob ignores $\left|\Psi_{1}\right\rangle$ to maximize his success probability $P_{b}$, when $P_{1}$ is less than the critical value $s^{2} /\left(s^{2}+t^{2}\right)$.

As is shown in Fig. 2, Bob's optimal success probability is enhanced by the deviation from equal probabilities. However, it can't reach 1 as $P_{1}$ approaches 0 . This fact is attributed to the requirement of no error in the task. As long as there is even a little probability of $\left|\Psi_{1}\right\rangle$, the value of $q_{2}^{b}$ is lower bounded by $s^{2} / t^{2}$ due to the overlap constraint.

After Bob's discrimination, the qubit $A$ is sent to the second observer Charlie, who knows Bob's protocol and performs a similar unambiguous discrimination. A necessary condition for Charlie's discrimination is that the states he receive are linearly independent 8, 18. In Eq. (11), there are four postmeasurement states sent to Charlie: $\left|\chi_{1}\right\rangle,\left|\chi_{2}\right\rangle,\left|\phi_{1}\right\rangle$ and $\left|\phi_{2}\right\rangle$. In the Hilbert space of the principal qubit, the independency requires $\left|\chi_{i}\right\rangle=\left|\phi_{i}\right\rangle$. Thus, the transformation in Eq. (1) should be

$$
U_{b}\left|\Psi_{i}\right\rangle\left|0_{b}\right\rangle=\left|\phi_{i}\right\rangle\left|\alpha_{i}\right\rangle_{b},
$$

where $\left|\alpha_{i}\right\rangle_{b}=\sqrt{q_{i}^{b}}|0\rangle_{b}+\sqrt{1-q_{i}^{b}}|i\rangle_{b}$ with $i=1,2$. One can find that, the absence of entanglement in the states (4) is a necessary condition of SSD. The task of Charlie is to distinguish the states $\left|\phi_{1}\right\rangle$ and $\left|\phi_{2}\right\rangle$ to extract the information encoded in $\left|\Psi_{1}\right\rangle$ and $\left|\Psi_{2}\right\rangle$ by Alice. Obviously, a necessary condition for his success is the overlap $\left\langle\phi_{1} \mid \phi_{2}\right\rangle=t<1$.

Similar to Eq. (4), Charlie makes a joint unitary operation $U_{c}$ between the qubit $A$ and his auxiliary qutrit $C$, with the parameters $q_{1}^{b}, q_{2}^{b}$ replaced by $q_{1}^{c}$ 
and $q_{2}^{c}$. The difference is that, his two postmeasurement states of qubit $A$ are the same, which indicates Charlie obtains all the information left by Bob. His optimal success probability is given by

$$
\begin{array}{ll}
\text { (i) : } \quad P_{c, \text { max }}=1-2 \sqrt{P_{1} P_{2}} t, & \text { when } \frac{t^{2}}{1+t^{2}} \leq P_{1} \leq \frac{1}{2} ; \\
\text { (ii) : } \quad P_{c, \text { max }}=P_{2}\left(1-t^{2}\right), & \text { when } 0<P_{1}<\frac{t^{2}}{1+t^{2}},
\end{array}
$$

corresponding to the values (i): $q_{1}^{c}=\sqrt{P_{2} / P_{1}} t$ and (ii): $q_{1}^{c}=1$ respectively.

When $P_{1} \neq 1 / 2$, although both the optimal success probabilities $P_{b \text {, max }}$ and $P_{c, \max }$ become piecewise functions, the former is a monotonous increasing function of the overlap $t$ and the later is a decreasing one. In other words, the trade-off relation between the information extracted by Bob and Charlie holds in the general case, which can be measured by $P_{b, \max }$ and $P_{c, \max }$ respectively.

One can obtain the success probability for both Bob and Charlie to identify the state as

$$
P^{S S D}=P_{1}\left(1-q_{1}^{b}\right)\left(1-q_{1}^{c}\right)+P_{2}\left(1-q_{2}^{b}\right)\left(1-q_{2}^{c}\right)
$$

Its maximum, for fixed $s$ and $P_{1} \leq 1 / 2$, occurs at $t=\sqrt{s}$ and $q_{1}^{b}=q_{1}^{c}$, which indicates the equivalence between the information extracted by Bob and Charlie. It is given by

$$
\begin{aligned}
& \text { (i) }: P_{\max }^{S S D}=\quad P_{1}\left(1-q^{*}\right)^{2}+P_{2}\left(1-\frac{s}{q^{*}}\right)^{2}, \quad \text { when } P_{C} \leq P_{1} \leq \frac{1}{2} ; \\
& \text { (ii) }: P_{\max }^{S S D}=\quad P_{2}(1-s)^{2}, \quad \text { when } 0<P_{1} \leq \min \left\{P_{C}, \frac{1}{2}\right\}
\end{aligned}
$$

where $q^{*}$ satisfies $P_{1} q^{* 4}-P_{1} q^{* 3}+P_{2} s q^{*}-P_{2} s^{2}=0$ and the critical value $P_{C}$ is determined by $P_{C}\left(1-q^{*}\right)^{2}+\left(1-P_{C}\right)\left(1-s / q^{*}\right)^{2}=\left(1-P_{C}\right)(1-s)^{2}$. For case (i), the optimal success probability occurs at $q_{1}^{b}=q_{1}^{c}=q^{*}$, while $q_{1}^{b}=q_{1}^{c}=1$ for case (ii), where Bob and Charlie conspire to ignore the state $\left|\Psi_{1}\right\rangle$.

When $s<3-2 \sqrt{2}, P_{C}<1 / 2$, it is similar to the problem to maximize $P_{b}$ or $P_{c}$ that the observers avoid the state with the lower probability. However, when $s \geq 3-2 \sqrt{2}, P_{C} \geq 1 / 2$, the case (i) vanishes. That is, even for the probabilities $P_{1}=P_{2}=1 / 2$, it is required to ignore one of the states in the optimal solution. The phenomenon is a symmetry breaking due to the lack of quantum information in qubit $A$ as pointed out in [8]. In Fig. 3, one can find that the optimal success probability $P_{\max }^{S S D}$ decreases with the overlap $s$, and increases with the deviation from the equal prior case. The region of case (i) is reduced by $s$ and the deviation. 
(a)

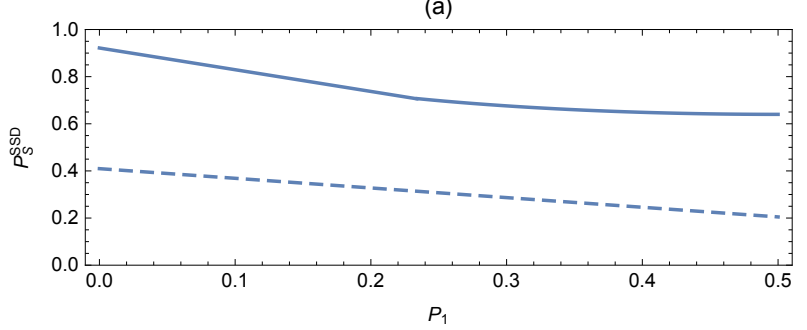

(b)

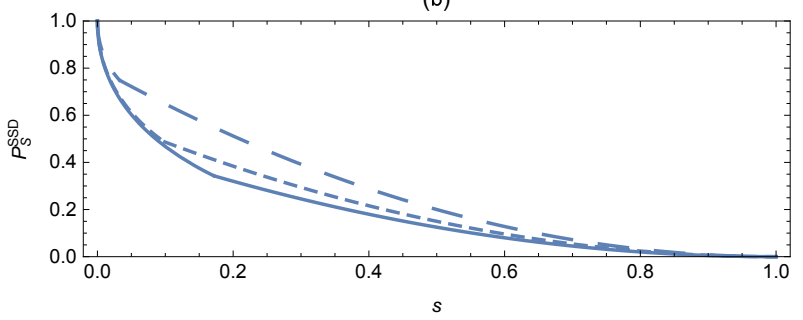

Fig. 3 The joint optimal success probability $P_{\max }^{S S D}$ as a function of the parameter $P_{1}$ and $s$ with the other fixed which are shown in Fig (a) and (b) respectively. (a): $s=0.04$ (solid line), 0.36 (dotted line); (b): $P_{1}=0.5$ (solid line), 0.4 (dotted line), 0.2 (dashed line).

\section{Comparison with other protocols}

In this part we compare the SSD with another three strategies that allow Bob and Charlie to communicate classically, which are studied in 22 with the equal prior probabilities.

(1) Bob performs an optimal unambiguous discrimination measurement on the qubit $A$, which requires $t=1$ in Eq.(3). He sends his results to Charlie through a classical channel. If Bob's outcome is "failure", they end the procedure. In the optimization, one need only consider the success of Bob, in distinguishing the two states with overlap $s$. Consequently, the maximal probability of both of them gaining the information sent by Alice is given by [12]

$$
\begin{array}{ll}
\text { (i) : } P_{\max }^{(1)}=1-2 \sqrt{P_{1} P_{2}} s, & \text { when } \frac{s^{2}}{1+s^{2}} \leq P_{1} \leq \frac{1}{2} ; \\
\text { (ii) : } P_{\max }^{(1)}=P_{2}\left(1-s^{2}\right), & \text { when } 0<P_{1}<\frac{s^{2}}{1+s^{2}} .
\end{array}
$$

These two cases require $q_{1}^{b}=\sqrt{P_{1} / P_{2}} s$ and $q_{1}^{b}=1$ respectively.

(2) Similar to the above task, Bob performs an optimal unambiguous discrimination measurement. If the outcome is "failure", he informs Charlie and end the procedure. Otherwise, he sends a qubit in the state he found to Charlie, and then Charlie performs an optimal unambiguous discrimination on the qubit. Here, we assume that Charlie knows the two states $\left|\Psi_{i}\right\rangle$ and their probabilities in Alice's preparation. 
In Appendix A, we show the details of optimization. The maximal probability for both Bob and Charlie to identify the state is a function divided in three cases as

$$
\begin{array}{lr}
\text { (i) }: P_{\max }^{(2)}=\left(1-2 \sqrt{P_{1} P_{2}} s\right)\left(1-2 \sqrt{P_{1}^{c} P_{2}^{c}} s\right), & \text { when } P_{c 1}<P_{1} \leq \frac{1}{2} ; \\
\begin{array}{lr}
\text { (ii) }: P_{\max }^{(2)}=\left(P_{2}-\sqrt{P_{1} P_{2}} s\right)\left(1-s^{2}\right), & \text { when } P_{c 2} \leq P_{1} \leq P_{c 1} ; \\
\text { (iii) }: P_{\max }^{(2)}=P_{2}\left(1-s^{2}\right), & \text { when } 0<P_{1}<P_{c 2},
\end{array}
\end{array}
$$

where the critical probabilities are

$$
\begin{aligned}
& P_{c 1}=\frac{s^{2}\left[s^{4}-\left(s^{2}-1\right) \sqrt{s^{4}-2 s^{2}+5}+3\right]}{2\left(s^{6}-s^{4}+3 s^{2}+1\right)}, \\
& P_{c 2}=\frac{s^{2}}{1+s^{2}},
\end{aligned}
$$

and $P_{i}^{c}=\left(P_{i}-\sqrt{P_{1} P_{2}} s\right) /\left(1-2 \sqrt{P_{1} P_{2}} s\right)$ with $i=1,2$. In case (i), $P_{1}^{c}$ and $P_{2}^{c}$ are the conditional probabilities given Bob's discrimination success of $\left|\Psi_{1}\right\rangle$ and $\left|\Psi_{2}\right\rangle$ received by Charlie. The parameters satisfy $q_{1}^{b}=\sqrt{P_{1} / P_{2}} s$ and $q_{1}^{c}=$ $\sqrt{P_{1}^{c} / P_{2}^{c}} s$. The value of $P_{1}^{c}$ decreases with the decreasing of $P_{1}$ from $1 / 2$, and always satisfies $P_{1}^{c} \leq P_{1}$. When $P_{c 2} \leq P_{1} \leq P_{c 1}$, the conditional probability of $\left|\Psi_{1}\right\rangle$ is less than the value of $P_{2}^{c}$. To maximize the total success probability, he only recognizes state $\left|\Psi_{2}\right\rangle$, which has a larger prior probability. The parameters satisfy $q_{1}^{b}=\sqrt{P_{1} / P_{2}} s$ and $q_{1}^{c}=1$. In case (iii), the optimal total probability requires that Bob ignores $\left|\Psi_{1}\right\rangle$, i.e. $q_{1}^{b}=1$. The conditional probability of $\left|\Psi_{1}\right\rangle$ is zero. Hence, once Charlie receives the qubit, he can learn the state in his hands being $\left|\Psi_{2}\right\rangle$. This fact makes optimal probability to be discontinuous at the point $P_{1}=P_{c 2}$.

(3) Bob performs a probabilistic unitary optimal clone operation on the qubit he receives from Alice 25,27. If Bob succeeds in cloning, he keeps one copy and sends the other one to Charlie. Then, Bob and Charlie perform optimal unambiguous discriminations to their respective qubits independently. While if Bob's cloning fails, he will inform Charlie and end the procedure.

The maximal probability of both of their succeeding is

$$
P_{\max }^{(3)}=P_{\max }^{\mathrm{cl}} P_{b, \max }^{\mathrm{cl}} P_{c, \max }^{\mathrm{cl}},
$$

where $P_{\max }^{\mathrm{cl}}$ is the maximal success cloning probability, and $P_{b, \max }^{\mathrm{cl}}=P_{c, \max }^{\mathrm{cl}}$ are the optimal success probabilities of the two discriminations. The form of $P_{b, \max }^{\mathrm{cl}}$ and $P_{c, \text { max }}^{\mathrm{cl}}$ are the same as Eq. (8), with the probabilities $P_{i}$ replaced by $P_{i}^{\mathrm{cl}}$. Here, $i=1,2$ and $P_{i}^{\mathrm{cl}}$ are the conditional probabilities of $\left|\Psi_{i}\right\rangle$ given Bob's cloning success, whose relations with $P_{i}$ and $s$ are given in Appendix B. The form of $P_{\max }^{\mathrm{cl}}$ and details to maximize the success probability in this protocol are shown in Appendix B

These results show that the joint optimal success probabilities of the above four protocols have more complicated properties than the special case with equal prior probabilities. In Fig. 4 one can find that as $P_{1}$ decreasing from $1 / 2$, 


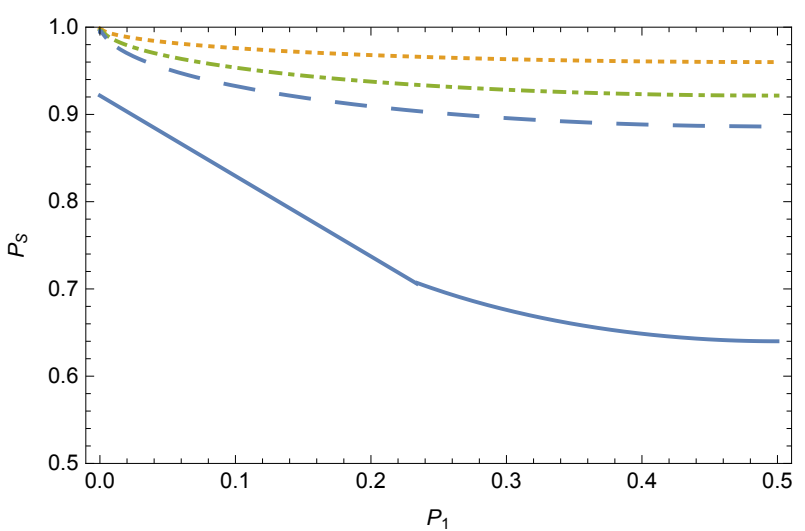

Fig. 4 The joint optimal success probability $P_{\max }$ as a function of $P_{1}$ is shown for $s=0.04$ corresponding to the four strategies respectively. Solid line: $P_{\max }^{S S D}$; dotted line: $P_{\max }^{(1)}$; dotdashed line: $P_{\max }^{(2)}$; dashed line: $P_{\max }^{(3)}$.

it becomes easier to extract the information sent by Alice in all the strategies. And, meanwhile, their differences decreases and the order remains unchanged.

Then we consider the probabilities $P^{*}$ that at least one of the observers succeeds in identifying the states, which are shown in Fig 5 It can be noticed that, the optimal probabilities of protocols (1), (2) and SSD are the same, where are $P_{\max }^{S S D *}=P_{\max }^{(1) *}=P_{\max }^{(2 *)}=P_{\max }^{(1)}$. But for the cloning protocol, the optimal probability is given by (see Appendix $\mathrm{C}$ for details)

$$
\begin{aligned}
& \text { (i) }: \quad P_{\max }^{(3) *}=P_{\max }^{\mathrm{cl}}\left(1-4 P_{1}^{\mathrm{cl}} P_{2}^{\mathrm{cl}} s^{2}\right), \quad \text { when } \frac{s^{2}}{1+s^{2}} \leq P_{1}^{\mathrm{cl}}<\frac{1}{2} \text {; } \\
& \text { (ii) : } \quad P_{\max }^{(3) *}=P_{\max }^{\mathrm{cl}}\left[1-\left(P_{1}^{\mathrm{cl}}+P_{2}^{\mathrm{cl}} s^{2}\right)^{2}\right], \quad \text { when } 0<P_{1}^{\mathrm{cl}}<\frac{s^{2}}{1+s^{2}} \text {. }
\end{aligned}
$$

As is shown in Fig. 15, these optimal success probabilities $P^{*}$ are enhanced by the deviation from equal prior probabilities. A difference with the existing results in 22 is that protocol (3) is superior to the other three strategies when $0<P_{1}<1 / 2$.

Above all, the deviation from equal prior probabilities makes it easier to gain the information sent by Alice. In the case with general prior probabilities, the strategies that allow communication all do better than SSD in the sense of both the observers gaining the information. In [22, this fact is ascribed to that SSD uses only one qubit while the others use more. This view is supported by our results when one considers at least one of the parties succeeds. Namely, as a new qubit is included after Bob's cloning in protocol (3), the discriminations of the two observers are independently from each other, while Charlie's success depends on Bob in the other three protocols. 


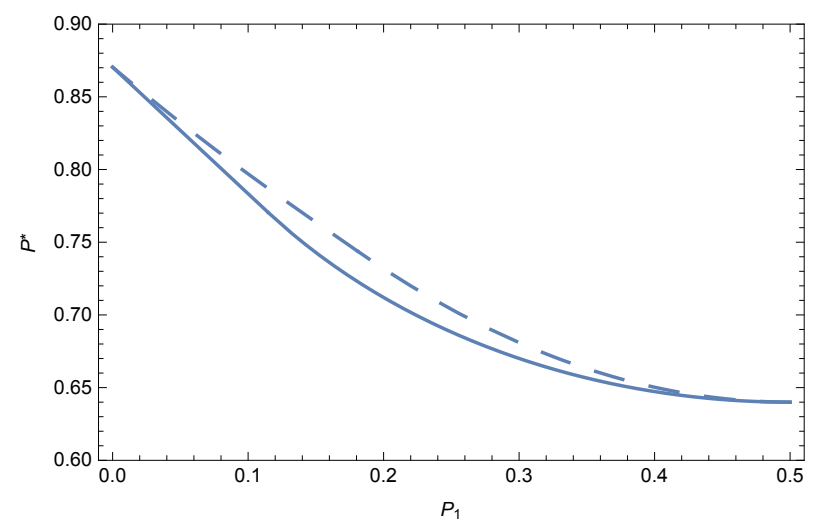

Fig. 5 The probability $P^{*}$ for one of the two parties succeeds as a function of $P_{1}$ for $s=0.36$. The four curves correspond to the four strategies respectively. Solid line: $P_{\max }^{S S D *}$, $P_{\max }^{(1) *}, P_{\max }^{(2) *}$; dashed line: $P_{\max }^{(3) *}$.

\section{Discords in SSD}

The key step in the process of discrimination is the joint unitary transformation between the system and ancilla in Eq.(4), followed by orthogonal measurements on the ancilla. This prompts us to examine roles of quantum correlations between the principal and the auxiliary systems in SSD with general non-uniform prior probabilities. Since entanglement is completely excluded by the form of Eq. (4), we focus on quantum discords in this part. The separable state in the discrimination of Bob can be written as

$$
\rho_{A B}=P_{1}\left|\phi_{1}\right\rangle\left\langle\phi_{1}|\otimes| \alpha_{1}\right\rangle_{b}\left\langle\alpha_{1}\left|+P_{2}\right| \phi_{2}\right\rangle\left\langle\phi_{2}|\otimes| \alpha_{2}\right\rangle_{b}\left\langle\alpha_{2}\right| .
$$

Discord is a kind of quantum correlation, which can exist in a separable state. It can be considered as the part of total correlation, measured by the quantum mutual information, which can be disturbed by the measurements on a subsystem 4. That is, there are two discords in state $\rho_{A B}$, corresponding to the measurements on subsystem $A$ or $B$. In the present work, we call the one with the measurements on $A$ as left discord and denote it as $D_{B A}$, while the other as right discord and $D_{A B}$.

The two discords of the two-rank system $\rho_{A B}$ can be derived by using the Koashi-Winter identity [28. Namely, one can consider $\rho_{A B}$ as a reduced state of the tripartite state

$$
|\Psi\rangle=\sqrt{P_{1}}\left|\phi_{1}\right\rangle\left|\alpha_{1}\right\rangle_{b}|0\rangle_{e}+\sqrt{P_{2}}\left|\phi_{2}\right\rangle\left|\alpha_{2}\right\rangle_{b}|1\rangle_{e},
$$

where $\left\{|0\rangle_{e},|1\rangle_{e}\right\}$ is the basis of a environment qubit $E$. Then, it is directly to obtain the residual tangle $\tau_{A B E}$ of the tripartite state and the tangles between one party with the other two as [29]

$$
\begin{array}{cc}
\tau_{A B E}=4 P_{1} P_{2}\left(1-t^{2}\right)\left(1-r^{2}\right), & \tau_{A \mid E B}=\tau_{A \mid B E}=4 P_{1} P_{2}\left(1-t^{2}\right), \\
\tau_{B \mid E A}=\tau_{B \mid A E}=4 P_{1} P_{2}\left(1-r^{2}\right), & \tau_{E \mid B A}=\tau_{E \mid A B}=4 P_{1} P_{2}\left(1-t^{2} r^{2}\right),(15)
\end{array}
$$


where we set $r=s / t=\left\langle\alpha_{1} \mid \alpha_{2}\right\rangle=\sqrt{q_{1}^{b} q_{2}^{b}}$. The right discord can be explicitly expressed as

$$
D_{A B}=H\left(\tau_{B \mid A E}\right)-H\left(\tau_{E \mid A B}\right)+H\left(\tau_{A \mid B E}-\tau_{A B E}\right),
$$

where

$$
H(x)=-\frac{1+\sqrt{1-x}}{2} \log _{2} \frac{1+\sqrt{1-x}}{2}-\frac{1-\sqrt{1-x}}{2} \log _{2} \frac{1-\sqrt{1-x}}{2} .
$$

The left discord $D_{B A}$ can be easily obtained by interchanging the subscripts $A$ and $B$ in Eq. (16). To analyze the roles of quantum discords in SSD, we define the proportion of left (right) discord in their total as

$$
\tilde{D}_{\text {left }}=\frac{D_{B A}}{D_{B A}+D_{A B}}, \quad \tilde{D}_{\text {right }}=\frac{D_{A B}}{D_{B A}+D_{A B}},
$$

and a symmetrized discord as

$$
D_{\text {symm }}=\sqrt{D_{B A} D_{A B}} .
$$

From the form of $\rho_{A B}$ in Eq. (13) and the relations in Eqs. (15) and (16), an obvious property can be noticed is that one can obtain $D_{B A}$ by exchanging $r$ and $t$ in $D_{A B}$. Consequently, $D_{\text {symm }}$ is symmetric under the exchanging of $r$ and $t$. Based on the symmetries and the curves in Fig. 6 we can check the conclusions in 8 and the influence of prior probabilities on discords.

As is shown in Fig. 6 (a), for fixed $P_{1}$ and $s$, the proportion of left discord $\tilde{D}_{\text {left }}$ increases with the overlap $t$. According to the mentioned symmetries, $\tilde{D}_{\text {right }}$ increases with $r$, and consequently decreases with $t$. Hence, the information extracted by Bob is positively correlated with $\tilde{D}_{\text {left }}$, and the information left to Charlie corresponds to $\tilde{D}_{\text {right }}$.

When $t=r=s^{1 / 2}$, the state $\rho_{A B}$ is symmetric under the permutation of $A$ and $B$. Thus, $\tilde{D}_{\text {left }}=\tilde{D}_{\text {right }}=1 / 2$ is independent of the prior probabilities. When $t>s^{1 / 2}>r, \tilde{D}_{\text {left }}>\tilde{D}_{\text {right }}$, and otherwise $\tilde{D}_{\text {left }}<\tilde{D}_{\text {right }}$. Fig. 6 (b) shows a curve in the former case, where $\tilde{D}_{\text {left }}$ is enhanced as $P_{1}$ moves away from $1 / 2$. According with the symmetry of $\rho_{A B}$ in Eq. (13), one can learn that the deviation increases the larger one while decreases the smaller one, and consequently enlarges their difference.

Fig. 66 (c) shows the symmetrized discord as a function of $P_{1}$ for different values of $t$. For fixed values of $P_{1}$ and $s$, the maximum symmetrized discord is reached at $t=r=s^{1 / 2}$, where occurs the optimal joint probability for both Bob and Charlie to identify the state. When $t=1$ or $s$, both the two discords $D_{A B}$ and $D_{B A}$ are zero as the state $\rho_{A B}$ becomes a product state. Thus, the discords are needed to realize the task of SSD. For fixed $t$, the value of $D_{\text {symm }}$, together with the difficulties of the two discriminations, are reduced by the deviation from the equal prior case. When $P_{1}$ approaches 0 or 1 , one of the two terms in $\rho_{A B}$ in Eq. (13) vanishes, and consequently both $D_{A B}$ and $D_{B A}$ become zero. 
(a)

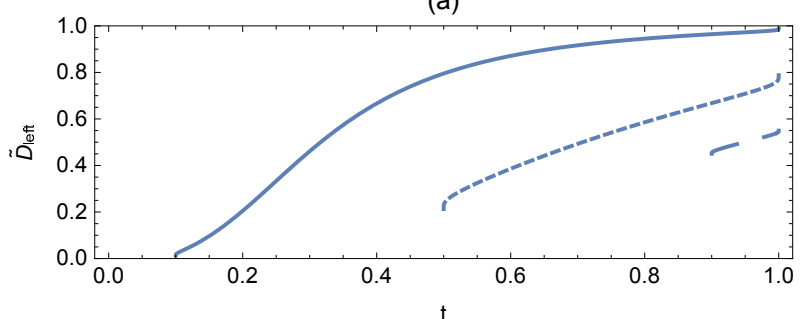

(b)

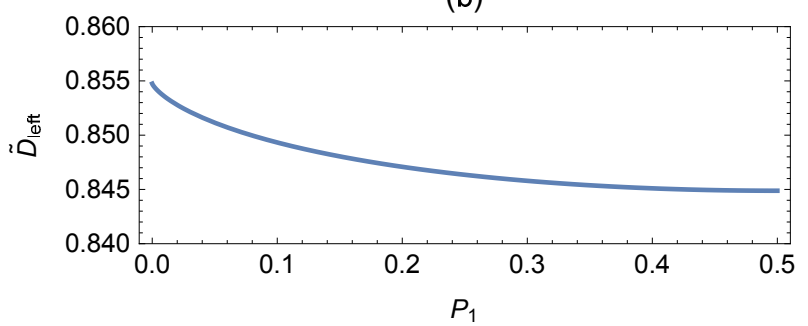

(c)

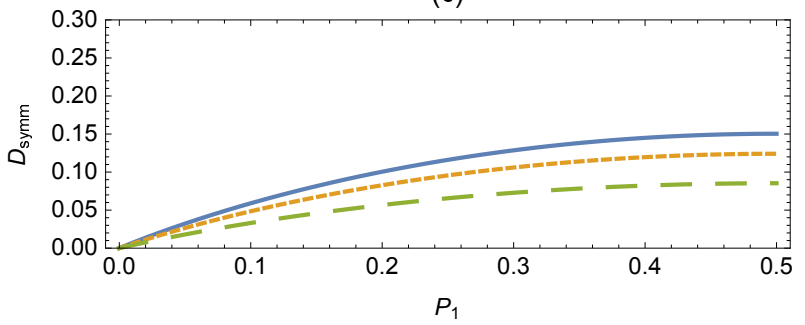

Fig. $6 \widetilde{D}_{\text {left }}$ as a function of the parameter $t$ and $P_{1}$ with the other variables fixed shown in (a) and (b). (a): $P_{1}=0.2, s=0.1$ (solid line), 0.5 (dotted line), 0.9 (dashed line); (b): $s=0.1, t=s^{1 / 4} ; \mathrm{Fig}(\mathrm{c})$ corresponds to the symmetrized discord as a function of the prior probability $P_{1}$ for $s=0.36, t=s^{1 / 2}$ (solid line), $s^{1 / 4}$ (dotted line) and $s^{1 / 8}$ (dashed line) respectively.

\section{Summary and Outlook}

The procedure of SSD in general case is investigated. We focus on the influence of prior probabilities on the properties found in the existing researches 8, 22. The deviation from equal probabilities represents more priori knowledge held by the observers before their measurements. It enhances the success probabilities in all tasks considered in the present work.

In the cases with general prior probabilities, the optimal success probabilities have more complicated details than the special cases with equal prior probabilities. In the sense of both the observers succeeding, all the three strategies that allow classical communication do better than SSD. This is consistent with the existing result, and is ascribed to that SSD uses only one qubit while the others use more in 22 . The imbalance of prior probabilities leads to that the protocol (3) is superior to others when one considers at least one of the 
parties succeeds. This result is an evidence of the mentioned viewpoint in 22, since a new qubit is introduced making the two discriminations in protocol (3) to be independent from each other.

The procedure for both Bob and Charlie to recognize the states requires the absence of entanglement in Bob's system-ancilla state. Quantum discords are necessary for their succeeding in discriminations. Both the left and right discords become zero when only one of the observers is allowed to gain the information, while their proportions in total are correlated with the information extracted by Bob and Charlie respectively. The symmetrized discord and joint success probability reach their maximums simultaneously when the left discord equals the right one. These conclusions are independent of the prior probabilities. The imbalance, corresponding to the priori knowledge of the observers, reduces the symmetrized discord but extends the relative differences between the left and right discords.

Our results may be generalized in several aspects. One of these is the SSD for $n(n \geq 3)$ nonorthogonal states, $\left|\psi_{i}\right\rangle$ (prepared with prior probabilities $P_{i}$, $i=0,1 \ldots n-1$ ), of a $n$ dimensional quantum system. Although, it is difficult to optimize the probability of success $P_{S S D}$, the conclusion about entanglement still hold true, which is required by the independency of the states sent to the second observer. That is, Bob's unitary transformation remains in the form of Eq. (4), and his system-ancilla state is

$$
\rho_{A B}=\sum_{i=0}^{n-1} P_{i}\left|\phi_{i}\right\rangle\left\langle\phi_{i}|\otimes| \alpha_{i}\right\rangle_{b}\left\langle\alpha_{i}\right|,
$$

where $\left\langle\phi_{i} \mid \phi_{j}\right\rangle\left\langle\alpha_{i} \mid \alpha_{j}\right\rangle=\left\langle\psi_{i} \mid \psi_{j}\right\rangle$. In addition, the ancilla is required to be a $2 n-1$ dimensional system [18. There are two extremes of Bob's transformation in Eq. (44) corresponding to zero discord [30, one of which is identity and another is a swap followed by a local unitary operation on the auxiliary system. In the former case, no information is extracted by Bob, and in the latter no information is sent to Charlie. This result is the same as the two-state case, shown in Fig. [6.

Another two generalizations is the extension to more than two consecutive observers and the one to mixed states. The optimizations in both cases are solved partly very recently [26]31, which are studied in POVM formalism. Besides analyzing the more general success probabilities, it is directly to describe these results via the Neumark formalism and study the roles of correlations in the procedures.

\section{Acknowledgment}

This work is supported by NSF of China (Grant No.11675119, No. 11575125, No.11105097). 


\section{References}

1. Horodecki, R., Horodecki, P., Horodecki, M., Horodecki, K.: Quantum entanglement. Rev. Mod. Phys. 81, 865-942 (2009)

2. Bell, J. S.: On the Einstein Podolsky Rosen paradox. Physics 1, 195-200 (1964)

3. Ollivier, H., Zurek, W. H.: Quantum Discord: A Measure of the Quantumness of Correlations. Phys. Rev. Lett. 88, 017901(2002)

4. Henderson, L., Vedral, V.: Classical, quantum and total correlations. J. Phys. A: Math. Gen. 34, 6899-6905 (2001)

5. Lanyon, B. P., Barbieri, M., Almeida, M. P., White, A. G.: Experimental Quantum Computing without Entanglement. Phys. Rev. Lett. 101, 200501 (2008)

6. Datta, A., Shaji, A., Caves, C. M.: Quantum Discord and the Power of One Qubit. Phys. Rev. Lett. 100, 050502 (2008)

7. Ekert, A K.: Quantum Cryptography Based on Bell's Theorem. Phys. Rev. Lett. 67, 661-663 (1991)

8. Pang, C.-Q., Zhang, F.-L., Xu, L.-F., Liang, M.-L., Chen, J.-L.: Sequential state discrimination and requirement of quantum dissonance. Phys. Rev. A 88, 052331 (2013)

9. Modi, K., Paterek, T., Son, W., Vedral, V., Williamson, M.: Unified View of Quantum and Classical Correlations. Phys. Rev. Lett. 104, 080501 (2010)

10. Bellomo, B., Giorgi, G. L., Galve, F., Lo Franco, R., Compagno, G., Zambrini, R.: Dynamics of geometric and entropic quantifiers of correlations in open quantum systems. Phys. Rev. A 86, 012312 (2012)

11. Roa, L., Retamal, J. C., Alid-Vaccarezza, M.: Dissonance is Required for Assisted Optimal State Discrimination. Phys. Rev. Lett. 107, 080401 (2011)

12. Zhang, F.-L., Chen, J.-L., Kwek, L. C., Vedral, V.: Requirement of Dissonance in Assisted Optimal State Discrimination. Sci. Rep. 3, 2134 (2013)

13. Peres, A.: How to differentiate between non-orthogonal states. Phys. Lett. A 128, 19 (1988)

14. Dieks, D.: Overlap and distinguishability of quantum states. Phys. Lett. A 126, 303-306 (1988)

15. Bennett, C. H.: Quantum Cryptography Using Any Two Nonorthogonal States. Phys. Rev. Lett. 68, 3121 (1992)

16. Bergou, J. A., Herzog, U., Hillery, M.: Quantum Filtering and Discrimination between Sets of Boolean Functions. Phys. Rev. Lett. 90, 257901 (2003).

17. Pang, S., Wu, S.: Optimum unambiguous discrimination of linearly independent pure states. Phys. Rev. A 80, 052320 (2009)

18. Roa, L., Retamal, J., Saavedra, C.: Quantum-state discrimination. Phys. Rev. A 66, $012103(2002)$

19. Chen, P.-X., Bergou, J. A., Zhu, S.-Y., Guo, G.-C.: Ancilla dimensions needed to carry out positive-operator-valued measurement. Phys. Rev. A 76, 060303(R) (2007)

20. Wu, X.-H., Gong, Y.-X.: Optical realization of the unambiguous discriminator for unknown quantum states. Phys. Rev. A 78, 042315 (2008).

21. Zhou, T.: Unambiguous discrimination between two unknown qudit states. Quantum. Inf. Process. 11, 1669-1684 (2012)

22. Bergou, J., Feldman, E., Hillery, M.: Extracting Information from a Qubit by Multiple Observers: Toward a Theory of Sequential State Discrimination. Phys. Rev. Lett. 111, 100501 (2013)

23. Nagali, E., Felicetti, S., de Assis, P.-L., Ambrosio, V. D., Filip, R., Sciarrino, F.: Testing sequential quantum measurements: how can maximal knowledge be extracted?. Sci. Rep. 2, 443 (2012)

24. Filip, R.: Coherent versus incoherent sequential quantum measurements. Phys. Rev. A 83, 032311 (2011)

25. Yerokhin, V., Shehu, A., Feldman, E., Bagan, E., Bergou, J. A.: Probabilistically Perfect Cloning of Two Pure States: Geometric Approach. Phys. Rev. Lett. 116, 200401 (2016).

26. Namkung, M., Kwon, Y.: Optimal sequential state discrimination between two mixed quantum states. Phys. Rev. A 96, 022318 (2017)

27. Duan, L.-M., Guo, G.-C.: Probabilistic Cloning and Identification of Linearly Independent Quantum States. Phys. Rev. Lett. 80, 4999-5002 (1998). 
28. Koashi, M., Winer, A.: Monogamy of quantum entanglement and other correlations. Phys. Rev. A 69, 022309 (2004)

29. Coffman, V., Kundu, J., Wootters, W. K.: Distributed entanglement. Phys. Rev. A 61, $052306(2000)$

30. Dakić, B., Vedral, V., Brukner, Č.: Necessary and Sufficient Condition for Nonzero Quantum Discord. Phys. Rev. Lett. 105, 190502 (2010)

31. Hillery, M., Mimih, J.: Sequential discrimination of qudits by multiple observers. J. Phys. A: Math. Theor. 50, 435301 (2017)

\section{A Calculations for protocol (2) that allows classical communication}

The optimization of success probability for both Bob and Charlie to succeed in identifying the state can be written as

$$
\begin{aligned}
& \operatorname{maximize}: P^{(2)}=\left[P_{1}\left(1-q_{1}^{b}\right)+P_{2}\left(1-q_{2}^{b}\right)\right]\left[P_{1}^{\prime}\left(1-q_{1}^{c}\right)+P_{2}^{\prime}\left(1-q_{2}^{c}\right)\right] \text {, } \\
& \text { subject to : } P_{i}^{\prime}=\frac{P_{i}\left(1-q_{i}^{b}\right)}{P_{1}\left(1-q_{1}^{b}\right)+P_{2}\left(1-q_{2}^{b}\right)}, i=1,2, q_{1}^{c} q_{2}^{c}=q_{1}^{b} q_{2}^{b}=s^{2}, \\
& \qquad q_{1}^{b}, q_{2}^{b}, q_{1}^{c}, q_{2}^{c} \in\left[s^{2}, 1\right], P_{1} \in(0,1 / 2] .
\end{aligned}
$$

The values of $P_{1}^{\prime}$ and $P_{2}^{\prime}$ are derived as

$$
\begin{aligned}
& \text { (i) : } P_{1}^{\prime}=P_{1}^{c}, P_{2}^{\prime}=P_{2}^{c}, \quad \text { when } \frac{s^{2}}{1+s^{2}} \leq P_{1} \leq \frac{1}{2} \text {; } \\
& \text { (ii) : } P_{1}^{\prime}=0, P_{2}^{\prime}=1, \quad \text { when } 0<P_{1}<\frac{t^{2}}{1+t^{2}} \text {. }
\end{aligned}
$$

The case (i) in Eq. 23. is divided into two subcases: (ia) $\frac{s^{2}}{1+s^{2}}<P_{1}^{\prime} \leq \frac{1}{2}$ and (ib) $0 \leq$ $P_{1}^{\prime} \leq \frac{s^{2}}{1+s^{2}}$ which correspond to the results in Eq. 9 $)$, (9b) respectively. The corresponding critical values $P_{c 1}$ in Eq. (9a) and Eq. (9) can be acquired after solving the equation which satisfy the successive boundary condition.

For case (ii) in Eq.23b), Bob gets optimized success probability for $q_{1}^{b}=1$. Then, for the next observer Charlie, the conditional probability is found to be $0\left(P_{1}^{\prime}=0\right)$ according to Eq. 222] and the state $\left|\psi_{1}\right\rangle$ is completely impossible to appear. Charlie can succeed in identifying the state with $100 \%$ probability because he has learned that his state is actually $\left|\psi_{2}\right\rangle$. Thus, the results in Eq. (9)) are obtained.

\section{B Calculations for protocol (3) where probabilistic cloning occurs}

Bob's unitary cloning operation is given by 25]

$$
\left.U\left(\left|\Psi_{i}\right\rangle\right)|0\rangle\right)=\sqrt{\gamma_{i}}\left|\Psi_{i}\right\rangle\left|\Psi_{i}\right\rangle\left|\lambda_{i}\right\rangle+\sqrt{1-\gamma_{i}}|\beta\rangle|\beta\rangle\left|\lambda_{0}\right\rangle, i=1,2,
$$

where $|0\rangle$ is a initialized state of the ancillas and $\left|\lambda_{i}\right\rangle,\left|\lambda_{0}\right\rangle$ are orthogonal states of the flag associated with successful cloning and failure cloning respectively. $\gamma_{i}$ is the success probability of the cloning for the state $\left|\Psi_{i}\right\rangle$ and $|\beta\rangle$ is a genetic failure state.

Thus we can get an optimized successful cloning probability as

$$
\text { maximize }: P^{\mathrm{cl}}=P_{1} \gamma_{1}+P_{2} \gamma_{2}
$$

subject to : $s=\sqrt{\gamma_{1} \gamma_{2}} s^{2}\left\langle\lambda_{1} \mid \lambda_{2}\right\rangle+\sqrt{\left(1-\gamma_{1}\right)\left(1-\gamma_{2}\right)}$. 
according to Eq. 24, where $\left|\lambda_{1}\right\rangle=\left|\lambda_{2}\right\rangle$ is required for optimal cloning 25.

If we set $\sin \theta_{i}=\sqrt{1-\gamma_{i}}(i=1,2)$ for $0 \leq \theta_{i} \leq \pi / 2$, the variables $x=\cos \left(\theta_{1}+\theta_{2}\right)$, $y=\cos \left(\theta_{1}-\theta_{2}\right)$ are further introduced. Eq. 26] is equivalent to $2 s=\left(1+s^{2}\right) y-\left(1-s^{2}\right) x$. And then we find an intermediate parameter $\omega$ which satisfies

$$
x=\frac{1-\left(1+s^{2}\right) \omega}{s}, y=\frac{1-\left(1-s^{2}\right) \omega}{s} .
$$

The range of the parameter $\omega$ is given in Eq. 31. It's found that

$$
\gamma_{i}=\frac{1}{2}\left[1+x y+(-1)^{i} \sqrt{\left(1-x^{2}\right)\left(1-y^{2}\right)}\right] .
$$

To seek the optimal value $P_{\max }^{\mathrm{cl}}$, the following equation should be satisfied $\left(P_{\max }^{\mathrm{cl}}\right)^{\prime}=$ $\frac{d P_{\max }^{\mathrm{cl}}}{d \omega}=0$. This equation is equivalent to $P_{1} \gamma_{1}^{\prime}+\left(1-P_{1}\right) \gamma_{2}^{\prime}=0$, thus the following results are obtained

where

$$
P_{1}=\frac{\gamma_{2}^{\prime}}{\gamma_{2}^{\prime}-\gamma_{1}^{\prime}}, P_{\max }^{\mathrm{cl}}=\frac{\gamma_{2}^{\prime} \gamma_{1}-\gamma_{1}^{\prime} \gamma_{2}}{\gamma_{2}^{\prime}-\gamma_{1}^{\prime}}
$$

here

$$
\gamma_{i}^{\prime}=\frac{d \gamma_{i}}{d \omega}=\frac{\sqrt{\gamma_{i}\left(1-\gamma_{i}\right)}}{s}\left[-\frac{1+s^{2}}{\sqrt{1-x^{2}}}+(-1)^{i} \frac{1-s^{2}}{\sqrt{1-y^{2}}}\right]
$$

And then, the conditional probabilities $P_{i}^{\mathrm{cl}}(i=1,2)$ of $\left|\Psi_{i}\right\rangle$ for the following two discriminations can be obtained as $P_{i}^{\mathrm{cl}}=\frac{P_{i} \gamma_{i}}{P_{1} \gamma_{1}+P_{2} \gamma_{2}}$. Hence, for the optimized successful cloning probability, $P_{i}, P_{i}^{\mathrm{cl}}, P_{\max }^{\mathrm{cl}}, P_{b, \max }^{\mathrm{cl}}$ and $P_{c, \text { max }}^{\mathrm{cl}}$ are all obtained as parametric functions of $\omega$ with the range

$$
\omega_{1} \leq \omega \leq \omega_{2}, \omega_{1}=\frac{1}{1+s}, \omega_{2}=\frac{1}{1+s^{2}},
$$

where $\omega_{1}$ and $\omega_{2}$ correspond to the cases for $P_{1}=P_{2}=\frac{1}{2}$ and $P_{1}=0$ respectively.

At last, the optimal success probability for both Bob and Charlie to identify the state is obtained as

$$
\begin{gathered}
\operatorname{maximize}: P^{(3)}=P_{\max }^{\mathrm{cl}}\left[P_{1}^{\mathrm{cl}}\left(1-q_{1}^{b}\right)+P_{2}^{\mathrm{cl}}\left(1-q_{2}^{b}\right)\right]\left[P_{1}^{\mathrm{cl}}\left(1-q_{1}^{c}\right)+P_{2}^{\mathrm{cl}}\left(1-q_{2}^{c}\right)\right] \\
\text { subject to }: q_{1}^{c} q_{2}^{c}=q_{1}^{b} q_{2}^{b}=s^{2}, q_{1}^{b}, q_{2}^{b}, q_{1}^{c}, q_{2}^{c} \in\left[s^{2}, 1\right]
\end{gathered}
$$

Thus, we can acquire the results in Eq.11 analytically.

\section{Optimal probability for at least one of Bob and Charlie succeeding in identifying the states}

It is obvious that the optimized probability $P_{\max }^{*}$ for one of their succeeding in discrimination for protocol (1) and (2) is equivalent to the results in Eq. 8). For SSD protocol, we can obtain the optimization as

$$
\text { maximize }: P^{S S D *}=P_{1}\left(1-q_{1}^{b} q_{1}^{c}\right)+P_{2}\left(1-q_{2}^{b} q_{2}^{c}\right)
$$

subject to $: P_{1} \in(0,1 / 2], q_{1}^{b} q_{2}^{b}=s^{2} / t^{2}, q_{1}^{c} q_{2}^{c}=t^{2}$,

$$
q_{1}^{b}, q_{2}^{b} \in\left[s^{2} / t^{2}, 1\right], q_{1}^{c}, q_{2}^{c} \in\left[t^{2}, 1\right] .
$$


Thus, this result is also equal to $P_{\max }^{(1)}$. For protocol (3), the maximal probability is derived

$$
\text { maximize : } P^{(3) *}=1-\left(P_{1}^{\mathrm{cl}} q_{1}^{b}+P_{2}^{\mathrm{cl}} q_{2}^{b}\right)\left(P_{1}^{\mathrm{cl}} q_{1}^{c}+P_{2}^{\mathrm{cl}} q_{2}^{c}\right)
$$

subject to $: q_{1}^{b} q_{2}^{b}=q_{1}^{c} q_{2}^{c}=s^{2}, q_{1}^{b}, q_{2}^{b}, q_{1}^{c}, q_{2}^{c} \in\left[s^{2}, 1\right], \quad P_{1} \in(0,1 / 2]$

Thus, the result in Eq. (12) can be easily obtained. 